\title{
Monitoring nuclear reactors with anti-neutrino detectors: the ANGRA project
}

\author{
Pietro Chimenti*ifor the ANGRA collaboration. \\ Universidade Federal do ABC-Brazil \\ E-mail: pietro.chimenti@ufabc.edu.br
}

\section{Ademarlaudo Barbosa}

Centro Brasileiro de Pesquisas Físicas

E-mail: laudoecbpf.br

\begin{abstract}
We describe the status of the ANGRA Project, aimed at developing an anti-neutrino detector for monitoring nuclear reactors. Indeed the detection of anti-neutrinos provides a unique handle for non-invasive measurements of the nuclear fluel. This kind of measurements are of deep interest for developing new safeguards tools which may help in nuclear non-proliferation programs. The ANGRA experiment, placed at about $30 \mathrm{~m}$ from the core of the $4 \mathrm{GW}$ Brazilian nuclear power reactor ANGRA II, is based on a water Cherenkov detector with about one ton target mass. A few thousand anti-neutrino interactions per day are expected. The latest results from simulations and the status of the construction are presented.
\end{abstract}

35th International Conference of High Energy Physics - ICHEP2010,

July 22-28, 2010

Paris France

\footnotetext{
* Speaker.

${ }^{\dagger}$ The author would like to thank the Brazilian agency FAPESP.
} 

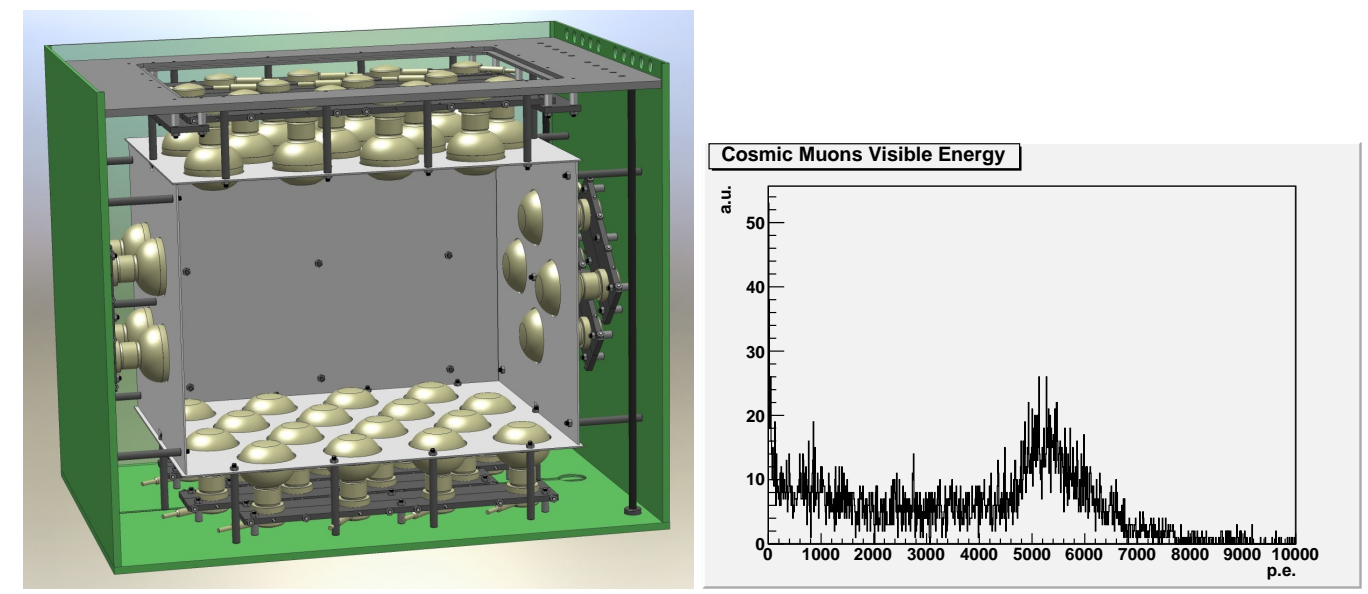

Figure 1: Left: Design of the Angra Water Cherenkov Detector (the external shield and veto is not included in the drawing). Right: expected distribution of photoelectrons produced by cosmic muons in the detector (simulation based on Geant4 [4]).

\section{The ANGRA project}

The possibility of applying antineutrino physics to monitor nuclear reactors for nonproliferation purpouses is of interest both of researchers and policymakers (see ref.[1] for an extended review). The ANGRA project [2] is currently developing a Gd-loaded Water-Cerenkov detector to monitor the the $4 \mathrm{GW}$ Brazilian nuclear power reactor ANGRA II.

The detector (Fig.1), with about 1 tonn fiducial volume, will be placed at about $27 \mathrm{~m}$ from the reactor core. In this condition a few thousand interaction per day of antineutrino are expected, to be compared with the rate of cosmic rays of about few hundred $\mathrm{Hz}$ (the detector will be placed at surface).

In order to reject this huge background a high efficiency veto will be placed outside the central detector. Moreover the signal in the detector due to cosmic rays is typically much higher in amplitude than the one due to antineutrinos (see Fig. 1 and Ref. [3] for a comparison with the antineutrino signal) allowing for a further discrimination.

Both the central Water-Cerenkov and the external veto are under construction and should be installed in 2011.

\section{References}

[1] A. Bernstein et al., "Nuclear Security Applications of Antineutrino Detectors: Current Capabilities and Future Prospects," arXiv:0908.4338 [nucl-ex].

[2] J. C. Anjos et al., "Angra neutrino project: Status and plans," Nucl. Phys. Proc. Suppl. 155 (2006) 231 [arXiv:hep-ex/0511059].

[3] P. Chimenti, M. A. Leigui de Oliveira and R. M. Lima [Angra Collaboration], "Measurements of neutrinos at the Angra reactor," PoS ISFTG (2009) 056.

[4] J. Allison et al., "Geant4 developments and applications," IEEE Trans. Nucl. Sci. 53 (2006) 270. 